\title{
A Phenomenological Theory of Neutron Irradiation Sensitivity of Ductile-Brittle Transition Temperature of Ferritic Steels*
}

\author{
By Naohiro Igata** and Ryukiti R. Hasiguti**
}

\begin{abstract}
Synopsis
The change of ductile-brittle transition temperature of ferritic steels due to neutron irradiation, $\Delta T_{\phi}$, which is called the irradiation sensitivity, was investigated and a phenomenological theory was presented. The theory predicts the change of sensitivity, $\delta\left(\Delta T_{\phi}\right)$, in terms of the changes of grain size, interstitial solute atom concentration, spacing of precipitates, and dislocation density.
\end{abstract}

\section{Introduction}

After neutron or other energetic particle irradiation, ferritic steels show hardening and embrittlement and their ductile-brittle transition temperatures shift to higher temperatures. This shift of transition temperature due to a given dose of irradiation, which we shall call the irradiation sensitivity, depends on microstructures of steels such as grain size, spacing of dispersed second phases, etc.

Theoretical investigations on the irradiation sensitivity have hitherto been rather scarce. Cottrell ${ }^{1)}$ and Nichols ${ }^{2)}$ made some theoretical treatments of the shift of transition temperature, but they did not consider the microstructure dependence of irradiation sensitivity.

In this paper, we shall present a phenomenological theory, by which we can understand the irradiation sensitivity of ferritic steels in terms of grain size, interstitial solute atom concentration, spacing of dispersed phases, and dislocation density.

\section{Theory of Irradiation Sensitivity}

Let us make the following assumptions :

(1) The ductile-brittle transition occurs at the intersecting point of the yield stress curve $* * *$ and the nil-ductility fracture stress curve ${ }^{* * *}$ as shown in Fig. 1. This is so-called Ludwig-Davidenkov criterion. ${ }^{3)}$

(2) The temperature dependence of the fracture stress $\sigma_{f}$ is small compared with that of the yield stress $\sigma_{y}$ so that the fracture stress is assumed, for simplicity, to be independent of temperature. ${ }^{4), 5) * * * *}$

(3) The change of the yield stress by irradiation at a given dose level $\phi$ will be expressed by $\Delta \sigma_{y \phi}$ which is assumed to be independent of temperature. $* * * *$

(4) The change of the fracture stress by irradiation

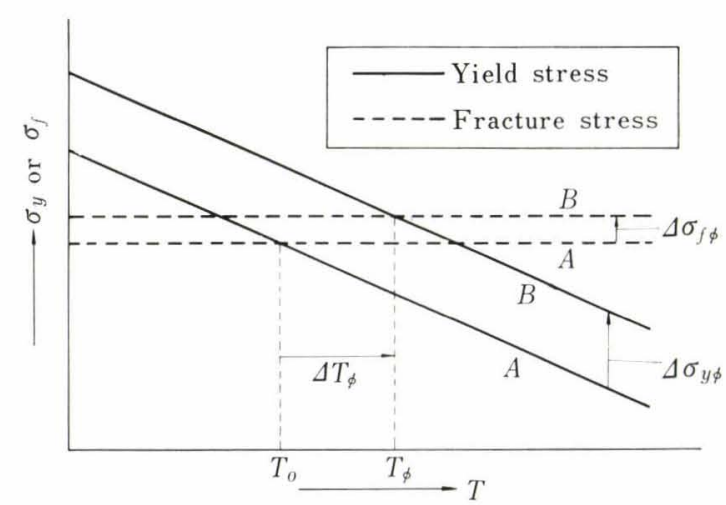

Fig. 1. Schematic diagram of yield stress and fracture stress as a function of temperature before $(A)$ and after $(B)$ irradiation. The fracture stress curves are drawn to conform to the assumption (2) in section $\boldsymbol{I I}$.

at a given dose level $\phi$ will be denoted by $\Delta \sigma_{f \phi}$ which is assumed to be independent of temperature. $* * * *$

Now in Fig. 1 the yield stress and the fracture stress are schematically shown as a function of temperature. After irradiation the yield stress curve and the fracture stress curve shift upward by an amount of $\Delta \sigma_{y \phi}$ and by an amount of $\Delta \sigma_{j \phi}$, respectively. If $\Delta \sigma_{y \phi}$ is larger than $\Delta \sigma_{f \phi}$, the ductile-brittle transition temperature, i.e., the intersection of the $\sigma_{y}$ curve and the $\sigma_{f}$ curve, shifts to a higher temperature, as shown in Fig. 1. Thus the shift of transition temperature due to a given dose of irradiation, i.e., the irradiation sensitivity, $\Delta T_{\phi}$, is expressed by

$$
\begin{aligned}
\Delta T_{\phi} & =T_{\phi}-T_{o} \\
& =\frac{\Delta \sigma_{f \phi}-\Delta \sigma_{y \phi}}{\Delta \sigma_{y} / \Delta T} \\
& =\frac{\Delta \sigma_{f \phi}-\Delta \sigma_{y \phi}}{d \sigma_{y} / d T}
\end{aligned}
$$

where $\mathcal{T}_{o}$ and $\mathcal{T}_{\phi}$ are the transition temperatures before and after irradiation, respectively.

\section{A. Grain Size Dependence of Irradiation Sensitivity}

Now let us assume that the yield stress and the frac-

* $\quad$ Received April 12, 1968.

** Department of Metallurgy, Faculty of Engineering, The University of Tokyo, Bunkyo-ku, Tokyo 113

*** "Fracture stress" means " nil-ductility fracture stress" in this paper, if not otherwise stated. The yield (fracture) stress curve means the yield (fracture) stress vs. temperature curve (Fig. 1), the yield (fracture) stress vs. grain size curve, etc.

**** The assumptions (2), (3), and (4), i.e., the assumptions of the temperature independent fracture stress, the temperature independent $\Delta \sigma_{f \dot{\phi}}$, and the temperature independent $\Delta \sigma_{y \dot{\rho}}$, are made for the sake of simplicity. These assumptions can be eliminated without changing essential conclusions of the theory, if the simplicity is sacrificed.

$* * * * *$ Equation (1b) which uses $d \sigma_{y} / d T$ instead of $\Delta \sigma_{y} / \Delta T$ is an approximate expression of (1a). Equation (1b) can only be used in the case where the yield stress curve can be approximated by a straight line. The case, where Eq. (1b) can not be used, will be described later elsewhere. 
ture stress are expressed by Petch's relations ${ }^{6), 7)}$ as follows :

$$
\begin{aligned}
& \sigma_{y}=\sigma_{o y}+k_{y} d^{-1 / 2} . \\
& \sigma_{f}=\sigma_{o f}+k_{f} d^{-1 / 2} .
\end{aligned}
$$

where $d$ is the grain diameter and $\sigma_{o y}, \sigma_{o f}, k_{y}$, and $k_{f}$ are the constants independent of $d$. We consider four parameters in this paper, i.e., the grain diameter, $d$, the interstitial solute atom concentration, $c$, the spacing of dispersed phases, $\lambda$, and the dislocation density, $\rho$. In Eqs. (2) and (3) $c$, $\lambda$, and $\rho$ are considered to be constants and are included in $\sigma_{o y}$ and $\sigma_{o f}$.

The grain size dependence of the irradiation sensitivity is obtained by differentiating Eq. (1) with respect to $\left(d^{-1 / 2}\right)$, considering Eqs. (2) and (3), i.e.,

$$
\begin{aligned}
& \frac{d\left(\Delta T_{\phi}\right)}{d\left(d^{-1 / 2}\right)} \\
= & \frac{\left(\Delta k_{f \phi}-\Delta k_{y \phi}\right)\left(\frac{d \sigma_{y}}{d T}\right)-\left(\Delta \sigma_{f \phi}-\Delta \sigma_{y \phi}\right)\left(\frac{d k_{y}}{d T}\right)}{\left(\frac{d \sigma_{y}}{d T}\right)^{2}}
\end{aligned}
$$

where

$$
\begin{aligned}
& \Delta \sigma_{y \dot{\phi}}=\Delta \sigma_{o y \phi}+\Delta k_{y \phi} d^{-1 / 2} \\
& \Delta \sigma_{f \dot{\phi}}=\Delta \sigma_{o f \phi}+\Delta k_{f \phi} d^{-12}
\end{aligned}
$$

with the constants $\Delta \sigma_{\text {oy } \dot{\phi}}, \Delta \sigma_{o f \phi}, \Delta k_{y \dot{\phi}}$, and $\Delta k_{f \phi}$.

In calculating Eq. (4), we have to consider the behaviors of $k_{y}$ and $k_{f}$ as functions of temperature and radiation dose, from which we can obtain the values of $\Delta k_{y \phi}, \Delta k_{f \phi}$, and $d k_{y} / d T$.

The behaviors of $k_{y}$ experimentally observed and found in literatures rather show diversity. For example, $k_{y}$ changes $^{8)-11)}$ or does not change ${ }^{8), 12)}$ with temperature; it decreases, $\left.{ }^{10)}, 13\right), 14$ ) increases, ${ }^{15)}$ or does not change ${ }^{16)}$ with irradiation. We shall, therefore, consider below various combinations of the above behaviors.

\section{(ai) The Case $A I$}

$k_{y}$ is independent of temperature and does not change with irradiation. In this case, we obtain from Eq. (4) the following result:

$$
\frac{d\left(\Delta T_{\phi}\right)}{d\left(d^{-1 / 2}\right)}=0
$$

Here the irradiation sensitivity does not depend on the grain size.

\section{(aii) The Case $A I I$}

$k_{y}$ is independent of temperature and decreases with irradiation $\left(\Delta k_{y \dot{\phi}}<0\right)$.* Now we get from Eq. (4)

$$
\frac{d\left(\Delta T_{\phi}\right)}{d\left(d^{-1 / 2}\right)}=-\frac{\Delta k_{y \dot{\phi}}}{d \sigma_{o y} / d T}
$$

$$
<0
$$

It may not be easy to check the result shown by Eq. (8) quantitatively, because the numerical data available now are too scarce to calculate the quantity given by Eq. (8). It may be convenient, therefore, to know the sign of Eq. (8). This is negative as shown by the relation (9), because $\Delta k_{y \phi}$ is negative as mentioned above and $d \sigma_{y} / d T$ is negative as shown schematically in Fig. 1 which results in a negative $d \sigma_{o y} / d T$ if $k_{y}$ is temperature independent. It should be pointed out that the negative value of Eq. (8) shows that the grain refinement is effective to reduce the irradiation sensitivity.

\section{(aiii) The Case $A I I I$}

$k_{y}$ depends on temperature and does not change with irradiation. In this case the grain size dependence of the sensitivity expressed by Eq. (4) will be

$$
\begin{aligned}
\frac{d\left(\Delta T_{\phi}\right)}{d\left(d^{-1 / 2}\right)}= & \frac{\left(\Delta \sigma_{y \dot{\phi}}-\Delta \sigma_{f \dot{\phi}}\right)}{\left(\frac{d \sigma_{y}}{d T}\right)^{2}} \cdot \frac{d k_{y}}{d T} \\
& <0 . \quad \ldots \ldots \ldots \ldots \ldots \ldots \ldots
\end{aligned}
$$

Now $d k_{y} / d T$ is negative, when $k_{y}$ is dependent on temperature. ${ }^{8)}$ As $\left(\Delta \sigma_{y \dot{\phi}}-\Delta \sigma_{f \dot{\phi}}\right)$ is usually considered to be positive, ${ }^{17)} \mathrm{Eq}$. (10) is usually negative. We see here again that the grain refinement is effective in reducing the irradiation sensitivity.

\section{(aiv) The Case $A I V$}

$k_{y}$ depends on temperature and decreases with irradiation $\left(\Delta k_{y \dot{\phi}}<0\right)$.** Now we obtain from Eq. (4)

$$
\frac{d\left(\Delta T_{\phi}\right)}{d\left(d^{-1 / 2}\right)}=\frac{-\Delta k_{y \dot{\phi}} \frac{d \sigma_{y}}{d \mathcal{T}}+\left(\Delta \sigma_{y \dot{\phi}}-\Delta \sigma_{f \dot{\phi}}\right) \frac{d k_{y}}{d T}}{\left(\frac{d \sigma_{y}}{d T}\right)^{2}} \ldots
$$

$<0$.

The relation (13) shows that the grain refinement in this case is also effective in reducing the irradiation sensitivity.

(av) The Case $A V$

$k_{y}$ depends on temperature and increases with irradiation $\left(\Delta k_{y \phi}>0\right){ }^{* *}$ In this case we get from Eq. (4)

$$
\frac{d\left(\Delta \mathcal{T}_{\dot{\phi}}\right)}{d\left(d^{-1 / 2}\right)}=\frac{-\Delta k_{y \dot{\phi}} \frac{d \sigma_{y}}{d T}+\left(\Delta \sigma_{y \dot{\phi}}-\Delta \sigma_{f \dot{\phi}}\right) \frac{d k_{y}}{d T}}{\left(\frac{d \sigma_{y}}{d T}\right)^{2}} .
$$

The sign of Eq. (14) is positive or negative as the sign of

$$
\left|\Delta k_{y \phi} \frac{d \sigma_{y}}{d T}\right|-\mid\left(\Delta \sigma_{y \dot{\phi}}-\Delta \sigma_{f \phi}\right) \frac{d k_{y}}{d T}
$$

\footnotetext{
* The temperature independent $k_{y}$ means the saturated or fully increased $k_{y}$ as a result of full aging. ${ }^{6)}$ It is unlikely that the saturated $k_{y}$ increases by irradiation. We consider, therefore, only the case where $k_{y}$ decreases by irradiation. (The case $A I I$ ).

** In the case of the temperature dependent $k_{y}, k_{y}$ can still increase. We shall, therefore, consider the increase as well as the decrease of $k_{y}$ by irradiation. (The cases $A I V$ and $A V$ ).
} 
is positive or negative. Thus the larger grains are effective in reducing $\Delta T_{\phi}$ if Eq. (14) is positive, and the refined grains are effective to reduce $\Delta T_{\phi}$ if Eq. (14) is negative.

\section{B. Solute Concentration Dependence of Irradiation Sensi- tivity}

Now we shall consider the effect of the interstitial solute atom concentration on the irradiation sensitivitity. We shall assume the following equation $^{4), 9), 18), 19 \text { ) }}$

$$
\sigma_{y}=\sigma_{o c}+k_{c} c^{m}
$$

where $c$ is the interstitial solute atom concentration and $\sigma_{o c}, k_{c}$, and $m$ are the constants. $m$ is $1 / 2^{18), 19)}$ or $1^{4), 9)}$ according to different authors. Here we keep $d$, $\lambda$, and $\rho$ constant, which are included in $\sigma_{o c}$.

The fracture stress $\sigma_{f}$ is assumed to be insensitive to the interstitial solute atom concentration for simplicity, so that we shall not provide an equation for $\sigma_{f}$ similar to Eq. (16).

$k_{c}$ in Eq. (16) is usually considered to be temperature dependent. $^{4)}$ But a result by Heslop and Petch ${ }^{9)}$ shows a temperature independent $k_{c}$, in which interstitial solute atoms seem to be in some precipitated conditions. We shall not consider, therefore, the temperature independent $k_{c}$.

It is assumed that $k_{c}$ may decrease, may increase, or may not change with irradiation. These three cases may all be possible according to the behaviors of radiation induced lattice defects which interact with solute atoms differently at different irradiation temperatures. Thus the following three combinations will be calculated.

\section{(bi) The Case BI}

$k_{c}$ is temperature dependent and does not change with irradiation. Then we get the derivative of Eq. (1) with respect to $c^{m}, d\left(\Delta T_{\dot{\varphi}}\right) / d\left(c^{m}\right)$, in a similar way to that of Eq. (4). Thus we obtain

$$
\frac{d\left(\Delta T_{\phi}\right)}{d\left(c^{m}\right)}=\frac{\left(\Delta \sigma_{y \phi}-\Delta \sigma_{f \phi}\right)\left(\frac{d k_{c}}{d T}\right)}{\left(\frac{d \sigma_{y}}{d T}\right)^{2}}
$$$$
<0 \text {. }
$$

The relation (18) is obtained because $d k_{c} / d T$ is negative. This shows that interstitial solute atoms (i.e., carbon or nitrogen) are effective in reducing the irradiation sensitivity.

\section{(bii) The Case BII}

$k_{c}$ is temperature dependent and decreases with irradiation $\left(\Delta k_{c \phi}<0\right)$. Now we get

$$
\begin{aligned}
\frac{d\left(\Delta \mathcal{T}_{\phi}\right)}{d\left(c^{m}\right)}=\frac{-\Delta k_{c \phi}\left(\frac{d \sigma_{y}}{d T}\right)+\left(\Delta \sigma_{y \dot{\phi}}-\Delta \sigma_{f \dot{\phi}}\right)\left(\frac{d k_{c}}{d T}\right)}{\left(\frac{d \sigma_{y}}{d T}\right)^{2}} \\
<0 . \quad \ldots \ldots \ldots \ldots \ldots \ldots \ldots \ldots \ldots \ldots \ldots \ldots \ldots \ldots
\end{aligned}
$$

The relation (20), which is easily obtained, shows again that interstitial solute atoms are effective in reducing the irradiation sensitivity.

\section{(biii) The Case BIII}

$k_{c}$ is temperature dependent and increases with irradiation $\left(\Delta k_{c \phi}>0\right)$. Then we obtain the same equation as Eq. (19). But as $\Delta k_{c \phi}$ is positive in this case, the sign of Eq. (19) changes as follows, i.e.,

$$
\frac{d\left(\Delta \mathcal{T}_{\phi}\right)}{d\left(c^{m}\right)} \gtrless 0
$$

according to the conditions

$$
\Delta k_{c \phi}\left(\frac{d \sigma_{y}}{d T}\right)|\lesseqgtr|\left(\Delta \sigma_{y \dot{\phi}}-\Delta \sigma_{f \phi}\right)\left(\frac{d k_{c}}{d T}\right) \mid
$$

respectively. The relations (21) and (22) show that the interstitial solute atoms are not always effective in reducing the irradiation sensitivity, if $\Delta k_{c \phi}$ is positive.

\section{Dependence of Irradiation Sensitivity on the Spacing of Dispersed Phases}

We shall consider the effect of the spacing of dispersed second phases such as carbides and nitrides on the irradiation sensitivity. Now we shall assume the equations

$$
\begin{aligned}
& \sigma_{y}=\sigma_{o y \lambda}+k_{y \lambda} \lambda^{-n} \\
& \sigma_{f}=\sigma_{o f \lambda}+k_{f \lambda} \lambda^{-n}
\end{aligned}
$$

where $\lambda$ is the spacing of the dispersed phases and $\sigma_{o y \lambda}, k_{y \lambda}, \sigma_{o f \lambda}, k_{f \lambda}$, and $n$ are the constants. $n$ is $1 / 2^{20)}$ or $1^{21)}$ according to different authors. Here we keep $d, c$, and $\rho$ constant, which are included in $\sigma_{o y \lambda}$ or in $\sigma_{o f \lambda}$. We shall consider some possible cases in the followings.

\section{(ci) The Case $C I$}

$k_{y \lambda}$ is independent of temperature and does not change with irradiation, and $k_{f \lambda}$ does not either change with irradiation. The derivative of $\mathrm{Eq}$. (1) with respect to $\lambda^{-1}, d\left(\Delta T_{\phi}\right) / d\left(\lambda^{-1}\right)$, is obtained in a similar way to that of Eq. (4). Then we get

$$
\frac{d\left(\Delta \mathcal{T}_{\phi}\right)}{d\left(\lambda^{-n}\right)}=0
$$

in this case. This means, of course, there is no effect of dispersed phases on the irradiation sensitivity.

\section{(cii) The Case $C I I$}

In deriving Eq. (1) in section $\boldsymbol{I I}$, it is assumed that the fracture stress increases by irradiation. But sometimes there occurs the case where the fracture stress decreases by irradiation. This occurs, for example, when the iron crystal contains precipitates of carbides or nitrides which are larger than a critical size. In this case the term $\Delta \sigma_{f \phi}$ in Eq. (1) should be replaced by $\left(-\Delta \sigma_{j \phi}\right)$, where the value of $\Delta \sigma_{f \phi}$ is kept always positive.

Now the conditions for the case CII are as follows. The fracture stress decreases by irradiation, so that we use the modified Eq. (26) instead of Eq. (1). This is

$$
\Delta \mathcal{T}_{\phi}=\frac{-\left(\Delta \sigma_{f \phi}+\Delta \sigma_{y \dot{\phi}}\right)}{\left(\frac{d \sigma_{y}}{d T}\right)}
$$


where $k_{y_{2}}$ is independent of temperature and does not change with irradiation, and $k_{f \lambda}$ decreases with irradiation $\left(\Delta k_{f \lambda \dot{\varphi}}<0\right)$. These conditions are sometimes actually observed in steels with coarse precipitates. ${ }^{22), 23)}$ Then we get the derivative of Eq. (26) with respect to $\lambda^{-n}$ as follows:

$$
\frac{d\left(\Delta \mathcal{T}_{\phi}\right)}{d\left(\lambda^{-n}\right)}=-\frac{\Delta k_{f \lambda \phi}}{\left(\frac{d \sigma_{y}}{d T}\right)}
$$

$<0$.

This shows that the dispersed phases are effective in reducing the irradiation sensitivity. In other words, the smaller the spacing, the less the shift of the transition temperature.

\section{(ciii) The Case CIII}

Here we use again Eq. (26) instead of Eq. (1). $k_{y \lambda}$ is independent of temperature and decreases with irradiation $\left(\Delta k_{y \lambda \phi}<0\right)$ and $k_{f \lambda}$ also decreases with irradiation $\left(\Delta k_{f, \phi}<0\right)$. Then we obtain

$$
\frac{d\left(\Delta \mathcal{T}_{\dot{\phi}}\right)}{d\left(\lambda^{-n}\right)}=-\frac{\Delta k_{y \lambda \dot{\phi}}+\Delta k_{f \lambda \dot{\phi}}}{\left(\frac{d \sigma_{y}}{d T}\right)}
$$

$$
<0 \text {. }
$$

This shows again that the dispersed phases are effective in reducing the irradiation sensitivity. Comparing the expressions (27) and (29), we can conclude that the case $C I I I$ is more effective than the case $C I I$ in reducing the sensitivity, if $\Delta k_{f \lambda \phi}$ in both cases are the same.

\section{Dependence of Irradiation Sensitivity on the Dislocation Density}

Now we shall consider the effect of dislocation density on the irradiation sensitivity. We assume the equations $^{24)}$

$$
\begin{aligned}
& \sigma_{y}=\sigma_{o y \rho}+k_{y \rho} \rho^{1 / 2} \\
& \sigma_{f}=\sigma_{o f \rho}+k_{f \rho} \rho^{1 / 2}
\end{aligned}
$$

where $\rho$ is the dislocation density and $\sigma_{o y \rho}, k_{y \rho}, \sigma_{o f \rho}$, and $k_{f \rho}$ are the constants. Here $d, c$, and $\lambda$ are kept constant and are included in $\sigma_{o y \rho}$ and $\sigma_{o f \rho}$. We shall consider the following one case.

\section{(d) The Case $D$}

$k_{y \rho}$ is independent of temperature and decreases with irradiation $\left(\Delta k_{y \rho \dot{\phi}}<0\right)$, while $k_{j \rho}$ does not change with irradiation. In this case we get the derivative of Eq. (1) with respect to $\rho^{1 / 2}$ as follows:

$$
\begin{aligned}
\frac{d\left(\Delta \mathcal{T}_{\dot{\phi}}\right)}{d\left(\rho^{1 / 2}\right)} & =-\frac{\Delta k_{y \rho \dot{\phi}}}{\left(\frac{d \sigma_{y}}{d T}\right)} \\
& <0 \quad \ldots \ldots \ldots . . .
\end{aligned}
$$

which shows that the increase in dislocation density is effective in reducing the irradiation sensitivity.

\section{Discussions}

Now let us examine the validity of Eq. (1) which is the basis of the present theory. In Table 1 the experimental values of $\Delta \sigma_{y \dot{\phi}}$ and $\Delta \sigma_{f \dot{\phi}}$, which are due to Trudeau, ${ }^{25)}$ are shown together with the average values of $\Delta \sigma_{y} / \Delta T$ between $-120^{\circ} \mathrm{C}$ and $0^{\circ} \mathrm{C}$, which are estimated from the experimental results of Rees, Hopkins, and Tipler. ${ }^{26)}$ The results of Trudeau are concerned with the binary alloys of iron, i.e., Fe-0.021\%$\mathrm{C}, \mathrm{Fe}-1.5 \% \mathrm{Mn}, \mathrm{Fe}-3.25 \% \mathrm{Ni}, \mathrm{Fe}-1.0 \% \mathrm{Cr}$, and $\mathrm{Fe}-$ $0.5 \%$ Mo. As the results of Rees et al. are not neces-

\begin{tabular}{|c|c|c|c|c|}
\hline Steel & $10^{18} \mathrm{nvt}^{* *}$ & $\begin{array}{c}\Delta \sigma_{y \phi^{25}}{ }^{25} \\
\left(\mathrm{~kg} / \mathrm{mm}^{2}\right)\end{array}$ & $\begin{array}{c}\Delta \sigma_{f \phi^{25)}} \\
\left(\mathrm{kg} / \mathrm{mm}^{2}\right)\end{array}$ & $\begin{array}{c}\left.\Delta \sigma_{y} / \Delta \mathcal{T}^{26}\right) \\
\left(\mathrm{kg} / \mathrm{mm}^{2} \cdot{ }^{\circ} \mathrm{C}\right)\end{array}$ \\
\hline \multirow{2}{*}{$\mathrm{Fe}-\mathrm{C}$} & 54 & 28.1 & 14.8 & 20.3 \\
\hline & 109 & 23.4 & 11.3 & 120 \\
\hline \multirow{3}{*}{$\mathrm{Fe}-\mathrm{Mn}$} & 55 & 32.8 & 19.5 & \multirow{2}{*}{16.3} \\
\hline & 72 & 29.1 & 15.8 & \\
\hline & 109 & 31.8 & 18.3 & 120 \\
\hline \multirow{4}{*}{$\mathrm{Fe}-\mathrm{Ni}$} & 68 & 34.8 & 24.4 & \multirow[b]{2}{*}{14} \\
\hline & 52 & 34.2 & 24.5 & \\
\hline & 60 & 33.6 & 23.4 & \multirow[t]{2}{*}{120} \\
\hline & 109 & 37.2 & 27.4 & \\
\hline \multirow{4}{*}{$\mathrm{Fe}-\mathrm{Cr}$} & 25 & 26.1 & 12.1 & \multirow[b]{2}{*}{19.6} \\
\hline & 52 & 27.5 & 13.5 & \\
\hline & 72 & 25.8 & 13.0 & \multirow[t]{2}{*}{120} \\
\hline & 110 & 29.5 & 17.4 & \\
\hline \multirow{2}{*}{ Fe-Mo } & 51 & 15.2 & 5.7 & 19.3 \\
\hline & 110 & 22.2 & 11.5 & 120 \\
\hline
\end{tabular}
sarily concerned with alloys of the same concentrations as those of Trudeau, the interpolations were made of the results of Rees et al.

Table 1. The change of yield stress $\Delta \sigma_{y \phi}$ and fracture stress $\Delta \sigma_{f \phi} *$ by irradiation and the average experimental temperature derivative of yield stress $\Delta \sigma_{y} / \Delta T$

* Here the tensile strength is used conventionally instead of fracture stress.

** Integrated neutron flux corresponding to $\Delta \sigma_{y}$ and $\Delta \sigma_{f} \phi$.

From these experimental values in Table 1 we can calculate $\Delta T_{\text {óal }}$ using Eq. (1). These values of $\Delta T_{\text {ocal }}$ are compared with the experimental values of $\Delta T_{\text {oexp }}$ obtained by Trudeau $^{25)}$ as shown in Fig. 2, where the abscissa shows $\Delta \mathcal{T}_{\text {gexp }}$ and the ordinate shows $\Delta T_{\text {ócal }}$. The values in Fig. 2 fall approximately on the $45^{\circ}$ straight line. This shows the validity of Eq. (1).

Now we shall discuss about the results of calculations described in section $\boldsymbol{I I}$. It is difficult at present to compare the calculated values of $d\left(\Delta T_{\phi}\right) / d\left(d^{-1 / 2}\right)$, $d\left(\Delta T_{\phi}\right) / d\left(C^{m}\right)$, etc. with the corresponding experimental values, because there scarcely are experimental results which were obtained under well specified conditions which can be quantitatively compared with the theory. What we can do at this moment is to compare the theoretical results qualitatively with experimental results. In other words we can discuss signs (i.e., plus or minus) of $d\left(\Delta T_{\phi}\right) / d\left(d^{-1 / 2}\right)$, etc. and compare them with experimental results. We shall describe below the 


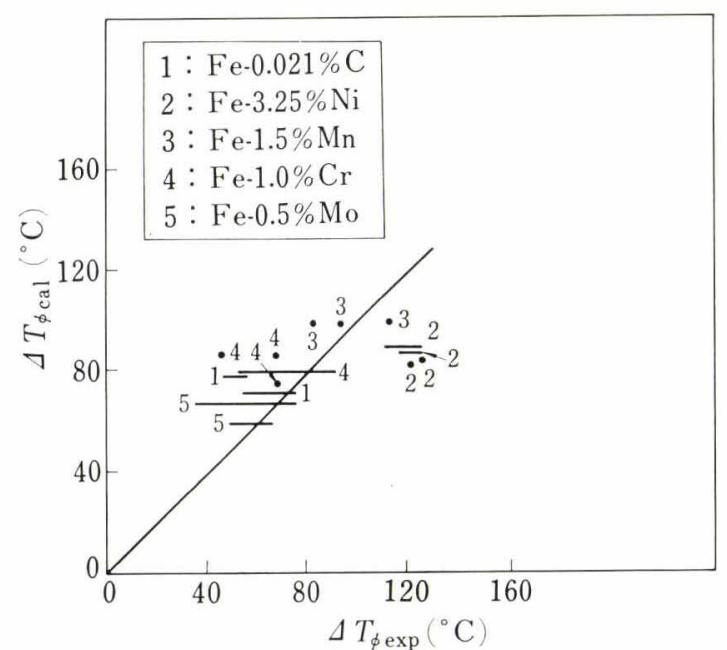

Fig. 2. The experimental irradiation sensitivity $\Delta T_{\phi_{\exp }} v s$. the calculated irradiation sensitivity $\Delta T_{\phi \text { cal }}$

cases where the experimental results are found in the literatures, with which the above mentioned signs of the irradiation sensitivity can be checked. It should be emphasized here that in all cases described below the experimental results agree well qualitatively, i.e., in sign, with the theory.

(1) If the steel is fully aged, namely if $k_{y}$ is independent of temperature, ${ }^{8), 12)}$ and if $k_{y}$ does not change with irradiation, then the grain refinement has no effect on the irradiation sensitivity ${ }^{27}$ ) (the case $A I$ : Eq. (7)). But if $k_{y}$ decreases with irradiation, the grain refinement reduces the irradiation sensitivity ${ }^{28)-32)}$ (the case $A I I$ : Eq. (9)).

The first case of the case $A V$, i.e., the case where the value (15) from Eq. (14) is positive, seems to be found in the reference, ${ }^{33)}$ where larger grains are effective in reducing the irradiation sensitivity.

(2) Two examples of the effects of interstitial solute atom concentrations are found in the literatures. In the case corresponding to $B I I$ (Eq. (20)) the higher solute concentration reduces the irradiation sensitivity ${ }^{10)}$ whereas in the case corresponding to the second case of BIII (Eq. (21)) the higher solute concentration increases $\Delta T_{\phi} \cdot{ }^{34)-36)}$

(3) If the steel contains precipitates of carbides or nitrides which are larger than a critical size, it occurs that the fracture stress decreases by irradiation. We find two cases concerning the effects of spacings between precipitations in the literatures, i.e., CII (Eq. (28)) and CIII (Eq. (30)). In both cases the smaller the spacing, the smaller the sensitivity. ${ }^{37), 38)}$

(4) If the dislocation density is increased, the irradiation sensitivity is decreased (the case $D$ : Eq. $(34))$. $^{39)}$

In this paper only the sensitivity, or the shift of the transition temperature, was discussed. But the transition temperature itself is affected by the metallurgical factors considered in this paper. This point will be discussed elsewhere later.

It is hoped that the irradiation experiments will be more extensively performed, in which metallurgical factors discussed in this paper will be more fully spe- cified and controlled. Then the present theory will be more thoroughly examined.

\section{REFERENCES}

1) A. H. Cottrell: Trans. Met. Soc. AIME, 212 (1958), 192.

2) F. A. Nichols: Phil. Mag., 14 (1966), 335.

3) See, for example, E. Orowan: Fracture, (1959), 147, John Wiley and Sons, New York; A. S. Tetelman and A. J. McEvily: Fracture of Structural Materials, (1967), John Wiley and Sons, New York.

4) N. P. Allen: Iron and Its Dilute Solid Solution, (1963), 297, Interscience Publishers, New York.

5) E. Orowan: Fatigue and Fracture of Metals, W. M. Murray ed., (1952), 139, Technology Press, MIT, Cambridge.

6) N. J. Petch: 7. Iron Steel Inst., 174 (1953), 25.

7) N. J. Petch: Phil. Mag., 1 (1956), 186.

8) R. M. Fisher: Ph. D. Dissertation, University of Cambridge, (1962), also cited by A. H. Cottrell: Proc. Conf. on the Relation between the Structure and Mechanical Properties of Metals, Teddington, (1963), 455, Her Majesty's Stationery Office, London.

9) J. Heslop and N. J. Petch: Phil. Mag., 1 (1956), 866.

10) J. G. Y. Chow, S. B. McRickard, and D. H. Gurinsky: BNL6249 (1963).

11) N.J. Petch: Fracture, Proc. Int. Conf. on the Atomic Mechanism of Fracture, Swampscott, (1954), 54, MIT, Cambridge.

12) H. Conrad and G. Schoeck: Acta Met., 8 (1960), 791.

13) A. A. Johnson: Phil. Mag., 5 (1960), 413.

14) D. B. Campbell and J. Harding: Proc. Conf. on the Response of Metals to High Velocity Deformation, Este Park, (1961), 51, Interscience Publishers, New York.

15) M. A. Adams and P. R. B. Higgins: Phil. Mag., 4 (1959), 777.

16) D. Hull and I. L. Mogford: Phil. Mag., 3 (1958), 1213.

17) L. F. Porter: ASTM, STP, No. 273 (1960), 147.

18) R. L. Fleischer and W. R. Hibbard, Jr.: Proc. Conf. on the Relation between the Structure and Mechanical Properties of Metals, Teddington, (1963), 261, Her Majesty's Stationery Office, London.

19) R. L. Fleischer: Acta Met., 11 (1963), 203.

20) G. S. Ansell and F. V. Lenel: Acta Met., 8 (1960), 612.

21) E. Orowan: Symposium on Internal Stresses, (1948), 451, Inst. Metals, London.

22) Japan Atomic Energy Research Institute, Report, No. 2847 (1967).

23 L. P. Trudeau: ASTM, STP, No. 273 (1960), 102.

24) A. Keh and S. Weissman: Electron Microscopy and Strength of Crystals, G. Thomas and J. Washburn, ed., (1963), 231, Interscience Publishers, New York.

25) L. P. Trudeau: Proc. Second Int. Conf. on the Peaceful Uses of Atomic Energy, (1958), Vol. 5, 475.

26) W. P. Rees, B. E. Hopkins, and H. R. Tipler: 7. Iron Steel Inst., 177 (1954), 93.

27) J. C. Wilson and R. G. Berggren: Proc. ASTM, 55 (1955), 689.

28) R. W. Nichols and D. R. Harries: ASTM, STP, No. 341 (1963), 162.

29) R. G. Berggren and J. C. Wilson: ORNL-CF-56-11-1 (1957).

30) A. T. Churchman, I. Mogford, and A. H. Cottrell: Phil. Mag., 2 (1957), 1271.

31) G. F. Carpenter, N. R. Knopf, and E. S. Byron: Nucl. Sci. Eng., 19 (1964), 18.

32) M. Grounes: The Effects of Radiation on Structural Metals, ASTM, STP, (1967); Aktiebolaget Atomenergi, Rep. S- 
350, Stockholm, (1966).

33) L. P. Trudeau: Special Rep. No. 69 (1961), 382, Iron Steel Inst., London.

34) R. L. Colomb, F. S. Rossi, and T. Sebbille: Solid State Commun., 4 (1966), 55.

35) M. Castagna et al.: The Effects of Radiation on Structural Metals, ASTM, STP, (1967).

36) F. S. Rossi and M. Castagna: EUR-3121 (1966), 391.
37) J. R. Hawthorne and L. E. Steele: 69th Ann. Meeting ASTM, Atlantic City, N. J., (1966).

38) E. Cibois, J. Lemaire, and M. Weiss: 6e Colloq. Met., (1962), 199.

39) R. R. Hasiguti, N. Igata et al.: to be published in Proc. 4th Int. Symp. on Effects of Radiation on Structural Metals, ASTM, (1968). 